\title{
Les pasteures et les mutations contemporaines du rôle du clerc
}

Jean-Paul Willaime

\section{(2) OpenEdition \\ Journals}

Édition électronique

URL : https://journals.openedition.org/clio/60

DOI : $10.4000 /$ clio. 60

ISSN : 1777-5299

Éditeur

Belin

Édition imprimée

Date de publication : 1 avril 2002

Pagination : 69-83

ISBN : 2-85816-620-X

ISSN : $1252-7017$

Référence électronique

Jean-Paul Willaime, "Les pasteures et les mutations contemporaines du rôle du clerc », Clio. Histoire, femmes et sociétés [En ligne], 15 | 2002, mis en ligne le 08 février 2005, consulté le 28 avril 2022. URL http://journals.openedition.org/clio/60 ; DOI : https://doi.org/10.4000/clio.60 


\section{Les pasteures et les mutations contemporaines du rôle du clerc}

Jean-Paul WiLLAIME

$\mathrm{Si}$, comme le souligne Raymond A. Mentzer, les Réformateurs protestants ont, au XVI siècle, contribué à renforcer le patriarcat familial en valorisant le pouvoir du mari dans le mariage et la famille ${ }^{1}$, reste qu'en plaçant dans l'unique autorité de la Bible, le lieu de la vérité religieuse, ils avaient potentiellement ouvert la voie d'une affirmation possible du pouvoir religieux des femmes. En faisant du recours à la Bible le principe suprême de la légitimité religieuse, les Réformes protestantes du XVI siècle (luthérienne, calviniste, zwinglienne, anabaptiste) avaient en fait érigé en institution un principe permanent de contestation de l'institution. Par l'affirmation du Sola Scriptura, la vérité religieuse devenait en effet un problème herméneutique qui ouvrit une discussion permanente sur l'interprétation des textes bibliques, en particulier au sujet des rôles respectifs de l'homme et de la femme. C'est l'impact de l'exégèse historico-critique des textes bibliques et le développement des sciences humaines qui, au XX⿳⺈ siècle, contribuèrent à faire sauter le verrou que constituaient les textes pauliniens pour des Églises revendiquant lautorité de la Bible². En 1964,

1 Mentzer 2001.

2 Pour l'histoire de l'accès des femmes au pastorat, nous nous permettons de renvoyer à nos deux études : Willaime 1996 et 1997.

CLIO, Histoire, Femmes et Sociétés, 2002, 15, pp. 69-83. 
dans les débats régionaux préparatoires au Synode National de l'Église Réformée de France de 1965 qui décida d'admettre pleinement les femmes au ministère de pasteur, le théologien André Dumas souligna ainsi que "l'on commettrait une faute grave en appliquant sans discernement à la femme d'aujourd'hui les recommandations que Paul adresse à la femme antique qui vivait dans des conditions totalement différentes»”.

\section{L'accès des femmes au pastorat}

Pour des Églises revendiquant l'autorité des Écritures, l'existence de textes bibliques limitant le rôle de la femme dans l'Église - particulièrement les textes pauliniens de 1 Corinthiens $14,34-35$ et 1 Timothée 2, 13-12 - ont longtemps constitué un puissant frein aux évolutions dans ce domaine. Tout en brouillant la distinction entre clerc et laïc et en fragilisant par là-même l'autorité du clerc, les Réformes protestantes aménageaient en fait un nouveau régime de pouvoir clérical : celui des pasteurs, considérés comme des "prédicateurs-docteurs" devant veiller à ce que la Bible soit lue et interprétée correctement, tant en doctrine qu'en pratique. Cette nouvelle forme de cléricature, celle des pasteurs, prétendait certes, au nom du sacerdoce universel des chrétiens, ne pas en être une : le pasteur était en effet considéré comme un laïc formé théologiquement et pouvant se marier ; un homme qui, bien qu'ordonné pour exercer certaines fonctions dans l'Église, partageait la condition du laïc et n'était pas fondamentalement différent de lui. Il reste que le pastorat constituait bien un rôle d'autorité, un rôle que l'on n'imaginait pas pouvoir confier à une femme, les mentalités du XVI siècle venant conforter les textes bibliques limitant la place de la femme. Mais si le protestantisme n'a pas immédiatement permis aux femmes d'accéder au rôle de pasteur, il a d'emblée introduit - ou, plutôt, historiquement, réintroduit - les femmes dans la proximité conjugale des clercs : Luther, comme Calvin, se marièrent.

3 Intervention d'André Dumas lors des débats des Synodes régionaux de 1964 ayant précédé le Synode National de 1965 (p. 232).

4 Nous avons précisé les spécificités du «clerc" protestant dans notre ouvrage Profession : pasteur. Sociologie de la condition du clerc à la fin du XX siècle, Genève, Labor et Fides, 1986, pp. 49-81. 
Avant l'apparition de la femme pasteur, c'est le rôle et l'image de la femme de pasteur qui, au cours de l'histoire, prévalurent : cette compagne pieuse et dévouée du pasteur qui participait à nombre d'aspects du ministère de son mari sans statut et reconnaissance ecclésiale particulière. C'est seulement en 1853, aux U.S.A., qu'une femme fut ordonnée pasteur dans une Église congrégationaliste. L'Armée du Salut accorda, dès sa fondation, une place importante aux femmes, de même que les quakers. De manière générale, la promotion des laïcs dans les mouvements de réveil et l'insistance sur l'engagement religieux personnel dans le protestantisme évangélique incitèrent les femmes à prêcher (par exemple, les femmes prédicatrices chez les quakers et dans le méthodisme américain à la fin du XVIII et au début du XIX' siècle) $)^{6}$.

$\mathrm{Au} \mathrm{XXc}$ siècle, des circonstances exceptionnelles comme les guerres ont favorisé, dans la vie ecclésiale comme dans la vie civile, la prise de responsabilités des femmes. Mais ces circonstances exceptionnelles n'étaient pas suffisantes pour asseoir définitivement le droit des femmes à exercer, dans toute sa plénitude, le ministère de pasteur. Les guerres relevaient encore de ces situations de crise qui, au cours de l'histoire, avaient déjà plusieurs fois permis la promotion du rôle des femmes. L'accès des femmes aux études de théologie fut un facteur beaucoup plus déterminant. Le protestantisme accordant une importance primordiale à la qualification théologique pour l'exercice d'une responsabilité religieuse, le fait que des femmes possèdent des diplômes de théologie pouvait leur ouvrir la porte du pastorat. Avant qu'il y eut des femmes pasteurs, il y eut des étudiantes en théologie et des diplômées en théologie. L'accès des femmes aux études de théologie s'est inscrit dans le mouvement général d'ouverture aux femmes des études universitaires; il fut facilité par l'existence de facultés de théologie d'État, relativement indépendantes des Églises. Ainsi, dès que les femmes commencèrent à accéder à l'Université allemande dans les premières années du XXc siècle, quelques étudiantes en théologie apparurent : la première à Marburg en 1909. En Suisse, des étu-

5 Reymond 1991.

6 Fath 2000. 
diantes en théologie protestante furent admises dès 1908 à Zurich et 1912 à Neuchâtel. En 1920, quatre jeunes filles étaient immatriculées à la Faculté de Théologie Protestante de Strasbourg. Cette présence de théologiennes dûment qualifiées interpella d'autant plus les Églises protestantes que, dans ces Églises, le pasteur est moins un médiateur du sacré qu'un théologien expliquant la Bible et édifiant les fidèles sur la base des Écritures. Compétentes théologiquement, les femmes pouvaient donc prétendre au ministère pastoral dans la plénitude de ses fonctions, même si toutes, au début, ne le souhaitaient pas forcément.

L'accès au pastorat ne se fit pas d'un seul coup. On parla tout d'abord de "ministères féminins", en se demandant en particulier si la spécificité des femmes ne devait pas les orienter vers des ministères spécialisés (aumôniers d'hôpitaux, de maisons des retraites, de mouvements de jeunesse). À Genève, en 1918, fut fondé un Institut des Ministères féminins qui prépara à l'exercice des fonctions d' "assistantes de paroisses" ou d' «aides-pasteurs". Ce n'est que progressivement que toutes les restrictions tombèrent et que les femmes eurent le même statut que leurs collègues masculins. L'accès à la pleine responsabilité pastorale et sans discriminations aucunes se fit donc très progressivement et par étapes successives. Schématiquement, on peut distinguer quatre étapes ; d'abord un «ministère féminin» à côté du ministère pastoral et d'un rang inférieur à lui ; puis un ministère centré sur certaines formes (non paroissiales) du pastorat ou sur certaines de ses dimensions (la catéchèse, la prédication dans certains cas et pour un public particulier : personnes âgées, auditoire féminin...) ; troisième temps, un ministère pastoral à part entière, mais à condition d'être célibataire ; enfin le ministère pastoral dans toutes ses attributions que la femme soit mariée ou non.

En 2001, en France, les femmes formaient $15 \%$ du corps pastoral de la Fédération Protestante de France (157 femmes sur un total de 1032 pasteurs). Mais dans les deux principales Églises de cette Fédération, l'Église Réformée de France et l'Église de la Confession d'Augsbourg d'Alsace et de Lorraine (luthérienne), les taux atteignaient respectivement, en 2001, 23 $\%$ et $20 \%$. À l'échelle mondiale, la grande majorité des Églises Luthériennes et Réformées admettent aujourd'hui le ministère pastoral des femmes. 
Selon des statistiques de 1992 produites par l'Alliance Réformée Mondiale - qui rassemble 177 Églises de tradition calviniste ou congrégationaliste de 87 pays -, seulement $25 \%$ des Églises membres de cette Alliance n'acceptaient pas encore l'ordination des femmes? ${ }^{7}$. Les femmes étant devenues pasteures, elles sont aussi amenées à ordonner d'autres femmes au ministère pastoral: ainsi, dès 1979 en Suisse alémanique (canton d'Argovie) une femme, la pasteure Sylvia Kolb consacrait-elle une autre femme, la pasteure Christine Nöthiger-Strahm. Les origines sociales des pasteures se sont diversifiées et la majorité d'entre elles sont mariées. On voit aussi de plus en plus apparaître des couples pastoraux, c'est-à-dire des couples où l'homme, comme la femme, sont pasteurs. Alors que certains pasteurs, dans les années qui suivirent l'accès des femmes au pastorat, évoquèrent le risque d'une forte féminisation de la profession - comme, par exemple, celle du corps enseignant -, on est loin du compte quarante ans après l'officialisation pleine et entière du ministère pastoral des femmes dans les Églises Luthériennes et Réformées de France. La possibilité pour les femmes de devenir pasteur ne s'est pas traduite par un raz de marée féminin même si la proportion de femmes parmi les pasteurs augmente lentement au fil des ans.

Accéder à un rôle longtemps réservé aux hommes est une chose, l'exercer en est une autre. À cet égard, il est nécessaire d'étudier la façon même dont les femmes pratiquent le ministère pastoral. Le pratiquent-elles comme les hommes ou ont-elles leur manière à elles de le pratiquer ? Si la réponse à cette question est positive, cela signifie-t-il que les femmes, en accédant au ministère de pasteur dans les Églises protestantes, contribuent à le transformer, à le faire évoluer ? Quelle est la condition des pasteures aujourd'hui et en quoi leur façon même d'assumer le pastorat et de l'exercer révèle-t-il certaines mutations du rôle de pasteur ? Telles sont les questions qui sont au centre de l'enquête quantitative et qualitative effectuée en 1995-1998 auprès des pasteures exerçant dans l'une ou l'autre Église rattachée à la Fédération Protestante de France ${ }^{8}$. La présente contribution

7 "Walk, my sister". The ordination of women: reformed perspectives, $1992: 165$.

8 Cette enquête, qui a été financée par l'Association des Pasteurs de France et qui s'est 
donne un premier aperçu des résultats de ces enquêtes en insistant sur le cadre général d'interprétation qui permet de les éclairer. La relative féminisation du pastorat participe en réalité d'une transformation plus générale de la pratique pastorale, une transformation allant dans le sens d'une décléricalisation encore plus marquée du ministère pastoral. La prise de responsabilité des femmes dans les Églises, au niveau ministériel, s'inscrit dans des évolutions plus générales concernant le religieux chrétien tel qu'il est vécu dans les sociétés occidentales actuelles, des évolutions qui engagent une redéfinition des rapports clercs/laïcs.

\section{Des femmes pasteurs : une seconde sécularisation du rôle du clerc}

C'est hypothèse centrale de notre recherche, après la première sécularisation du rôle de clerc qu'a représenté la Réformation du XVI siècle ellemême. Le protestantisme représente une étape importante dans le mouvement de sécularisation interne du christianisme, une sécularisation interne qu'au XX⿳亠丷厂 siècle, un sociologue comme François-André Isambert a bien repérée dans l'évolution même du catholicisme post-conciliaire'. L'émergence du pastorat, au XVI siècle, représente incontestablement une certaine sécularisation du clerc, une sécularisation caractérisée par le passage d'un pouvoir sacré à un pouvoir intellectuel et moral. Avec le pasteur protestant en effet, le clerc n'est plus un personnage sacré jouissant d'un statut ontologique autre, il est un homme parmi les autres et peut donc se marier et vivre en famille comme tout laïc. Cette première sécularisation contribua à réintégrer le clerc dans la société et la vie quotidienne. Mais l'important magistère intellectuel et moral exercé par le pasteur, ajouté au fait que tout pouvoir sacré n'avait pas disparu - notamment à travers le monopole de l'administration des sacrements du baptême et de la sainte cène -, limita les effets de cette première sécularisation. Les

déroulée dans les années 1995-1998, a été double : une enquête quantitative par questionnaire et une enquête qualitative par entretiens semi-directifs. Un ouvrage, en préparation, présentera l'analyse détaillée des résultats de ces enquêtes et les réflexions auxquels ils peuvent donner lieu. L'objectif étant d'analyser les mutations contemporaines du rôle de pasteur à la lumière de la situation et du vécu des femmes pasteurs.

9 Isambert 1976. 
femmes ont d'abord été admises à prêcher avant d'être admises à administrer les sacrements (réduits à deux dans le protestantisme : le baptême et la sainte cène).

Cette réticence accentuée à propos de l'administration des sacrements par des femmes s'inscrit, il est important de le souligner, dans une réticence plus générale manifestée par rapport à la prise en charge des sacrements par des laïcs. Dans l'enquête de 1978-1979 auprès des pasteurs de France, j'avais été frappé par le fait que si $54 \%$ des pasteurs interrogés étaient prêts à confier la prédication à un laïc, seulement $37 \%$ étaient prêts à laisser un laïc présider la sainte cène. Or, si prendre en charge la prédication exige des savoirs et des compétences, la présidence de la sainte cène ne demande pas de formation spécifique. La pasteure Martine Millet, en 1995, témoignait pour sa part de la réticence manifestée par certains à l'égard d'enterrements effectués par des femmes : "les enterrements, c'est le lieu où les femmes pasteurs ont le plus de difficultés quand elles arrivent dans une paroisse. On trouve que la femme n'est pas faite pour enterrer, on préfere un homme, c'est clair. C'est un lieu de tension qui interroge les gens. Moi, j’ai été renvoyée puis, d'autres fois, j'étais la seule, alors il fallait faire avec». Le pasteur protestant, aussi désacralisée que soit la figure de clerc qu'il incarne, est toujours pour nombre de personnes celle ou celui qui médiatise du sacré. Face à cette dimension, on observe, dans les Églises protestantes comme dans d'autres milieux religieux, certaines réticences quand il s'agit de femmes. L'accès des femmes au pastorat peut donc, selon nous, être vu comme une seconde sécularisation du rôle du clerc, une seconde sécularisation caractérisée par la perte de pouvoir du clerc et la dissolution de son statut d'exception. L'accès des femmes s'inscrit dans cette seconde étape, c'est-à-dire que la féminisation du pastorat est constitutive de ce processus, même si ce processus, qui touche aussi les hommes pasteurs, ne s'y réduit pas. Quels sont les éléments indiquant cette sécularisation plus poussée du clerc et dans quelle mesure l'accès des femmes au pastorat $\mathrm{y}$-a-t-il contribué ?

L'évolution de la figure du pasteur, du "prédicateur-docteur" à «l'animateur-écoutant», le passage d'un rôle d'autorité à celui d'accompagnateur, d'un rôle d'enseignant à celui de communicateur et médiateur est un 
premier aspect important. Cette évolution profonde, que nous avions déjà relevée dans notre enquête auprès des pasteurs ${ }^{10}$, contribue à banaliser encore plus le pastorat, à en faire quelque chose comme une profession sociale à dimension spirituelle. Si c'était l'image de l'enseignant qui, les siècles précédents, se rapprochait le plus de celle du pasteur, c'est aujourd'hui celle de l'animateur social ou du psychothérapeute "conseiller de vie" (Lebensberater, dit-on en Allemagne). Ce faisant, le pastorat se rapproche de la vie quotidienne. C'est beaucoup moins le pasteur-évêque, maître en doctrine et gardien de la morale, que le laïc théologien qui accompagne les autres laïcs dans leur recherche de sens et leur quête de repères éthiques. Or, c'est au moment même où le pastorat évolue d'un rôle didactique et autoritaire à un rôle d'écoute et d'accompagnement que les femmes y accèdent en nombre ; au moment donc où le pastorat se rapproche d'une profession sociale, centrée sur la relation et l'animation. On constate, à propos du pastorat, ce que l'on observe dans d'autres professions, en particulier les professions de l'enseignement et de la santé : uneféminisation relative au moment même où cette activité a perdu de son prestige social et où elle se banalise. Les femmes deviennent pasteures alors même que le pastorat se "féminise" par son statut social et les fonctions qu'il remplit. Nous mettons des guillemets pour souligner qu'il s'agit ici d'un stéréotype selon lequel une profession se "féminiserait" en perdant du pouvoir - ce qui revient à associer genre masculin et rôle de pouvoir - et en se focalisant sur des activités d'écoute et de relation, activités réputées, toujours selon ce stéréotype, plus «féminines». En ce sens, il est clair que les hommes participent tout autant à cette "féminisation" du pastorat. Dans une autre enquête effectuée en 1988 auprès des 25 pasteures de l'E.C.A.A.L. et de l'E.R.A.L., Anne Muller-Heitzmann constate que les femmes ne veulent pas perpétuer l'image du pasteur «autoritaire, paternal et austère» et souhaitent être "au milieu du groupe et pas audessus». Les femmes pasteurs disent qu'elles «désaustérisent» le ministère pastoral "1. Plusieurs des témoignages apportés en 1995 par des pasteures à

10 Willaime 1986.

11 Muller-Heitzmann 1988. 
la session sur «la condition pastorale au miroir des femmes» organisée par l'Association des Pasteurs de France ${ }^{12}$ vont dans le même sens : les femmes pasteurs valorisent elles-mêmes les aspects relationnels, d'accompagnement et d'écoute du ministère pastoral et ont tendance à minorer la dimension d'autorité de ce ministère. Si les femmes se sentent particulièrement en affinité avec cette façon d'assumer le ministère pastoral, la proportion des femmes pasteurs pourrait continuer à augmenter sensiblement, d'autant plus que cette façon de voir le ministère pastoral correspond aujourd'hui aux attentes des laïcs à l'égard des clercs.

\section{Professionnalisation et déplacement de pouvoir}

Cette sécularisation du pastorat s'inscrit dans une professionnalisation du rôle pastoral qui se manifeste tout particulièrement par le fait qu'on tend plus à identifier le pastorat à un faire qu'à un être, à une pratique sociale qu'à un statut. La distinction entre vie professionnelle et vie privée s'accentue et les pasteurs ne se considèrent plus pasteurs 24 heures sur 24 (insistance sur le jour hebdomadaire de congé et sur les vacances). C'est la fin du presbytère : le logement même du pasteur se privatise et se distingue des locaux paroissiaux et du bureau qu'il y occupe. Les temps de congé et de loisirs sont mieux distingués des temps de travail et les pasteurs reçoivent sur rendez-vous. En mettant en avant son rôle de mère et en demandant des congés pour les périodes de maternité, la pasteure mariée accentue cette distinction vie privée/vie professionnelle (alors que la pasteure célibataire, au contraire, peut quelquefois les assimiler, la paroisse devenant, comme déclare l'une d'entre elles, "sa famille»). De fait, nombre de femmes pasteurs, ayant de réelles difficultés à articuler leur métier de pasteur et ses horaires spécifiques (réunions en soirée, activités le week-end) avec leurs responsabilités familiales - d'autant plus que les foyers pastoraux continuent à donner une assez nombreuse progéniture - décident de se mettre en congé pour une durée variable. Sur soixante pasteures dans l'E.R.F. en 1991, douze étaient ainsi en congé (dont dix

12 Cabiers de l'Association des Pasteurs de France, $\mathrm{n}^{\circ}$ 26, Septembre 1995 : 4-53. 
mariées avec des pasteurs) ${ }^{13}$. Le fait d'exercer le pastorat par intermittence, les périodes de congés de maternité ou pour convenances personnelles succédant à des périodes d'activités, tend en tout cas à séculariser encore plus le ministère pastoral, à en faire une pratique professionnelle comme une autre et non plus un statut lié à un engagement à vie.

Alors qu'au début, nombre de pasteures étaient célibataires, ce taux a très nettement diminué aux générations suivantes : en 1991, dans l'Église Réformée de France, sur 60 pasteures, on dénombrait 15 célibataires (soit $25 \%$ ) et, sur les 45 autres mariées, 27 l'étaient avec un pasteur. En 1995, sur l'ensemble des pasteures exerçant en France dans l'une des Églises rattachées à la Fédération Protestante de France, $23 \%$ étaient célibataires et $30 \%$ étaient mariées avec un pasteur. On observe ainsi une forte croissance du nombre de couples pastoraux, ce qui ne va pas sans entraîner divers problèmes, notamment financiers. À côté des pasteures célibataires et des couples pastoraux, on a aussi de plus en plus, en France, le cas de pasteures mariées avec des époux occupant une bonne position professionnelle (ingénieur, architecte, comptable, professeur...). En 1995, $23 \%$ des pasteures étaient mariées avec des cadres supérieurs ou professions libérales. Une évolution sensible s'est faite dans la situation socio-professionnelle des couples où l'un des conjoints est pasteur : on passe du couple homme-pasteur / femme au foyer ou femme-enseignante, infirmière ou assistante sociale (cas typique du couple pastoral dans les années soixante$\mathrm{dix}^{14}$ ) au couple homme-ingénieur / femme-pasteure comme on est passé du couple homme-instituteur ou professeur / femme au foyer au couple homme-cadre / femme institutrice ou professeure de collège. Le pastorat

13 Enquête de Arina Van de Kerk auprès de "ministres, mères de jeunes enfants" dans l'Église Réformée de France, 1991. L'auteur insiste également sur les difficultés financières : la majorité des femmes pasteurs mères de jeunes enfants «dépensent la plus grande partie de leur rémunération pour la garde des enfants. Certaines parlent de deux tiers, d'autres de trois quarts, d'autres d'opération blanche !n.

14 Willaime 1986 : 99-100; en 1978-1979, les épouses de pasteurs exerçaient ou avaient exercé surtout les professions d'infirmière (ou autre profession para-médicale) : $16 \%$, d'institutrices : $13 \%$, de secrétaires : $11 \%$, d'assistante sociale (ou autre profession sociale) : $9 \%$. 
devient dans ce cas une profession d'appoint dans le couple, les principaux revenus étant assurés par le mari dans une profession séculière.

Il y a d'autre part un déplacement de pouvoir, une ségrégation verticale qui s'effectue dans le partage du pouvoir religieux. D'un côté les "grands clercs», ceux que nous appelons les «théologiens de société», qui élaborent leur discours et leur action en vis-à-vis avec la société globale, tentant d'y assurer la place du christianisme et de répondre aux défis contemporains. Ceux aussi qui occupent les positions dirigeantes dans l'appareil ecclésiastique. De l'autre, les clercs de proximité qui gèrent la religion dans la vie quotidienne au plus près du vécu et des préoccupations des gens. Le pastorat n'échappe pas à cette dualisation de la religion et l'on peut se demander si l'accès des femmes au pastorat ne participe pas, de façon privilégiée, à cette religion de proximité, humaine et chaleureuse, qui se met en place alors même que le pouvoir religieux s'est déplacé en se focalisant beaucoup plus sur les responsabilités ecclésiastiques (ce qui permet d'exercer, même de façon très souple et dialoguée, un certain pouvoir sur les autres pasteurs) et sur la gestion du face-à-face avec la société notamment à travers les medias. La féminisation du pastorat serait congruente avec la demande sociale d'une religion apprivoisée, une religion du vécu, près du foyer, tendances auxquelles les hommes n'échappent pas eux-mêmes. Quant à la religion sociétale, celle qui concerne la place et le rôle de la religion dans le système institutionnel de la société globale, elle reste plus investie par les hommes pasteurs, même si quelques femmes s'y investissent aussi. Bien qu'une femme pasteur ait été présidente d'Église (Thérèse Klipffel, qui fut Présidente de l'E.R.A.L. de 1982 à 1988) et que certaines d'entre elles accèdent à des fonctions de responsabilité ecclésiastique (Marie-France Robert est ainsi devenue en 200 "Inspecteur ecclésiastique" dans l'Église Évangélique Luthérienne de France), les pasteures sont encore sous-représentées dans les responsabilités ecclésiastiques supérieures. Par contre, la forte attention médiatique dont elles sont l'objet dans un pays de culture catholique comme la France assure à quelques-unes d'entre elles une visibilité sociale certaine.

Les verbatim de la trentaine d'entretiens semi-directifs qui ont été effectués en 1997 auprès de pasteures exerçant en France dans l'une des 
Églises de la Fédération Protestante de France sont extrêmement riches et l'analyse détaillée de ces entretiens figurera dans une prochaine publication. Annonçons d'ores et déjà ici qu'ils confirment que si, dans la phase de conquête du ministère pastoral par les femmes, la distinction masculin/féminin était effacée (ou utilisée par les pasteurs hommes pour s'opposer au ministère pastoral des femmes), les pasteures d'aujourd'hui revendiquent sans complexe une spécificité féminine dans la façon même d'exercer leur ministère. Les résultats de cette enquête confirment assez bien les constats faits par Janine Mossuz-Lavau et Anne de Kervasdoué dans leur ouvrage Les femmes ne sont pas des hommes comme les autres (1997). Les pasteures insistent particulièrement sur la distinction entre leur vie professionnelle au service d'une Église et leur vie privée, et ce aussi bien dans l'espace (logement) que dans le temps (congé, vacances). Bien que ces tendances à la professionnalisation et à la sécularisation du rôle pastoral soient également observables chez les hommes, les femmes les accentuent; de même que leur insistance sur l'écoute et le relationnel dans l'exercice du ministère pastoral. C'est un Dieu de proximité, proche des gens et de la vie quotidienne que les femmes pasteurs veulent signifier. Remettant en cause l'image du Dieu Tout Puissant et de la théologie qui est sous-jacente à cette représentation, certaines pasteures privilégient un Dieu faible. On découvre dans les plis de certains discours un autre rapport au divin et une autre façon de se positionner comme "professionnel du religieux». Ces résultats confirment plusieurs des remarques faites par Martine Millet dans l'interview accordée à Florence Rochefort et publiée dans Clio 2/1995 : "Je crois que le ministère pastoral exercé par des femmes est un peu plus proche des gens. Le problème des femmes pasteurs, c'est souvent d'ailleurs de savoir comment garder une certaine distance. J'ai une amie qui détestait prêcher du haut de la chaire, elle s'était approchée des gens et on le lui a reproché, alors elle a mis la robe noire pour mettre la distancem. Diverses spécificités se dégagent également de la situation des couples pastoraux (où les deux conjoints sont pasteurs). L'examen de ces entretiens permet également de déceler les difficultés rencontrées par telle ou telle pasteure : contrairement à ce qu'une certaine image médiatique de la femme pasteur indique, les femmes pasteurs ne 
sont pas toutes satisfaites de leur condition et quelques-unes éprouvent des difficultés réelles à combiner leur vie familiale avec leur rôle de pasteure.

L'accès des femmes au pastorat s'inscrit dans des mutations profondes du pastorat lui-même, dans ce qu'il devient dans la pratique. Dans le protestantisme, il participe d'une sécularisation encore plus poussée du rôle clérical, une sécularisation qui s'accompagne d'un déplacement de la différenciation sexuelle pour le partage du pouvoir. Quant à la revendication d'égalité comme thème ayant joué un rôle important dans l'accès des femmes au pastorat, elle a certes joué un rôle, mais il est intéressant de constater que ce ne sont pas les femmes intéressées par le ministère pastoral qui ont été les plus militantes dans ce domaine. Ce sont les femmes de pasteurs, plus que les femmes pasteurs ou candidates au pastorat qui ont été les plus en pointe dans les combats pour les droits des femmes. Si les femmes ont conquis le rôle de pasteur et si, ce faisant, elles ont effectivement mis les femmes et les hommes à égalité devant le ministère pastoral, ce n'est pas prioritairement au nom d'une revendication d'égalité et d'aspirations féministes, mais au nom de leur compétence théologique et parce qu'elles désiraient s'engager au service d'une Église. Dans le catholicisme, on peut se demander si l'accès des femmes à la prêtrise ne contribuerait pas à ébranler tout un édifice hiérarchique en faisant du catholicisme un peu plus une religion de laïcs. Dans l'accès des femmes aux responsabilités sacerdotales, c'est aussi la sécularisation interne du christianisme qui est en jeu. C'est bien parce que le protestantisme a été un vecteur essentiel de cette sécularisation qu'il a aussi été le premier, parmi les confessions chrétiennes, à admettre les femmes au pastorat. 


\section{Bibliographie}

ALTHAUSEN Gudrun, BERLIS Angela, BUSCH Christine et alii, 2000, Kinder, Kirche und Karriere. Von der berufstätigen Mutter im Dienst des Herrns. Erfahrungsberichte, Berlin, Wichern-Verlag.

BLOCHER-SAILLENS Madeleine, 1961, Libérées par Christ pour son service, Nogent-sur-Marne, édité par l'auteur.

Cabiers de l'Association des Pasteurs de France, $\mathrm{n}^{\circ}$ 26, septembre 1995 : Pastorale Nationale Villebon-sur-Yvette (Essone) des 5-7 février 1995 sur «La condition pastorale au miroir des femmes».

DUMAS André, 1965, Église Réformée de France, LVIIK Synode National, Nantes, 30 avril-3 mai 1965, Cahors, I.F.Q.A.

FATH Sébastien, 2000, «La prédication féminine en protestantisme évangélique», Hokhma, 74/2000, pp. 23-60.

ISAMBERT François-André, 1976, «La sécularisation interne du christianisme", Revue Française de Sociologie, XVII, 4, pp. 573-589.

LAUTMAN Françoise éd., 1997, Ni Ëve ni Marie. Luttes et incertitudes des héritières de la Bible, Genève, Labor et Fides.

MENTZER Raymond A., 2001, «La place et le rôle des femmes dans les Églises Réformées", Archives de Sciences Sociales des Religions, 113, janvier-mars, pp. 119-132.

MILLET Martine, 1992, "Le ministère pastoral des femmes dans le protestantisme français", in Jean Delumeau, La religion de ma mère. Le rôle des femmes dans la transmission de la foi, Paris, Cerf, pp. 343-362.

—, 1995, Interview de Martine Millet, pasteur de l'Église Réformée de France, par Florence Rochefort (Paris, le 15 juin 1995), Clio, 2, Presses Universitaires du Mirail, pp. 341-349.

MOSSUZ-LAVAU Janine, de KERVASDOUE Anne, Les femmes ne sont pas des hommes comme les autres, Paris, Editions Odile Jacob, 1997. MULLER-HEITZMANN Anne, 1988, La condition des Femmes Pasteurs en Alsace-Moselle, Mémoire de maîtrise, Faculté de Théologie Protestante, Université des Sciences Humaines de Strasbourg, 1988. 
PARMENTIER Elisabeth, 1998, Les filles prodigues. Défis des théologies féministes, Genève, Labor et Fides.

REYMOND Bernard, 1991, La femme de pasteur. Un sacerdoce obligé ? Genève, Labor et Fides.

ROUVERAND Edith, 1990, Marieleine Hoffet ou Les combats d'une femme-pasteur en Alsace de 1905 à nos jours, Strasbourg, Éditions Oberlin.

SAMMET Kornelia (unter Mitarbeit von Annette Wilkes), 1998, Beruf: Pfarrerin. Eine empirische Untersuchung zu Berufsbild und Berufspraxis von Pfarrerinnen in der Evangelischen Kirche in Berlin-Brandenburg, Berlin, Berlin Verlag, Arno Spitz GmbH.

SCHMIDT Elisabeth, 1976, En ces temps de malheur... J'étais pasteur en Algérie. 1958-1962, Paris, Cerf.

-, 1978, Quand Dieu appelle des femmes. Le combat d'une femme pasteur. Préface d'André Dumas, Paris, Cerf.

"Walk, my sister». The ordination of women : reformed perspectives, Studies from the World Alliance of Reformed Churches, $\mathrm{n}^{\circ}$ 18, Genève, 1992.

WILLAIME Jean-Paul, Profession : pasteur. Sociologie de la condition du clerc à la fin du XX' siècle, Genève, Labor et Fides, 1986.

—, "L'accès des femmes au pastorat et la sécularisation du rôle du clerc", Archives de Sciences Sociales des Religions, 95, juillet-septembre 1996, pp. 29-45.

—, «Les femmes pasteurs en France : socio-histoire d'une conquête», in Françoise Lautman éd., Ni Ėve ni Marie. Luttes et incertitudes des héritières de la Bible, Genève, Labor et Fides, 1997, pp. 121-140. 